\title{
Histological composition of lumbar disc herniations related to the type of herniation and to the age
}

\author{
Matveeva N, Zivadinovik J, Zdravkovska M, Jovevska S, Bojadzieva B \\ Insitute for Anatomy, Faculty of Medicine, University "St. Kiril and Metodij", Skopje, R. of Macedonia. \\ nikimatveeva@gmail.com
}

\begin{abstract}
Objectives: To compare the data for histologic composition of the herniated disc material between different types of disc herniations and between patients of different age.

Background: Lots of studies have investigated the histologic composition of disc herniations. Few studies have examined the presence of granulation tissue related to the type of herniations and age of the subjects.

Methods: 120 patients divided in to two age groups underwent MR imaging before microsurgical removal of the herniations. Disc herniations were divided in to two groups, non migrated and large migrated disc herniations. The histologic assessment of the herniated material is done by dividing the intercellular matrix into four types. The existence of areas of granulation tissue and hyaline cartilage is evaluated too.

Results: The tissue composition showed significant differences between patients of different age. Areas of granulation tissue and neovascularisation are found in $12 \%$ of 64 protrusions and contained extrusions, and in $29 \%$ of 56 large migrated disc extrusions $(p=0.029)$. In elderly patients the tissue composition changed with a significant decrease of prevailing nucleus pulposus composition in $7 \%$ of $60(p=0.000)$ and a significant increase of prevailing fibrous tissue composition in $40 \%$ of 60 patients $(p=0.000)$. Areas of granulation tissue and neovascularisation were found in $27 \%$ of 60 patients aged $\leq 50$ and in $13 \%$ of 60 patients aged $>50$.

Conclusions: The tissue composition of the herniated material showed more expressed differences between subjects of different age. Granulation tissue and neovascularisation were more frequent findings in large migrated disc herniations and in patients aged 35 to 50 years (Fig. 8, Ref. 22). Full Text in PDF www.elis.sk.

Key words: magnetic resonance (MR), spine, lumbar disc herniations, histology.
\end{abstract}

Disc herniations are a common cause of low back pain and sciatica. It is well known that the majority of lumbar disc herniations diagnosed at magnetic resonance imaging recover spontaneously (1). Lots of authors reported spontaneous decrease in the size of herniated masses (2). According to Saal more than $50 \%$ of the herniations show reduction in $82 \%$ of patients (3). There are also numerous studies for the benefits of surgical treatment of disc herniations with high primary success rate (4). Although there are some absolute indications for operative treatment of disc herniations like cauda equine syndrome and severe, progressive neurologic deficits, it is not clear yet which therapeutic management should be preferred. According to Takada sequestered hernia and transligamentous extrusions seem to be more rapidly absorbed (5). The exposure of these types of disc herniations to epidural vascular supply provokes inflammatory and immune reactions and phagocytosis. These types of disc herniations regress in size rapidly, so conservative treatment should be preferred in these cases. Some au-

Insitute for Anatomy, Faculty of Medicine, University „St. Kiril and Metodij“, Skopje, R. of Macedonia

Address for correspondence: N. Matveeva, "Ruzveltova" 4/35, 1000 Skopje, R. of Macedonia

Phone/Fax: +389.2.3125304

Acknowledgement: We would like to express our gratitude to Prof. M. Vrcakovski from the Institute for Radiology (University Clinical Center,Skopje) for his support and cooperation. thors reported that differences in the histologic composition of the herniated disc material may be in relation to different inflammatory responses (6). The presence of granulation tissue and neovascularisation in the histologic composition of the herniated disc material indicate that a natural healing mechanism is activated, which may lead to a rapid spontaneous regression of the disc herniation. On the other side, some authors reported that presence of hyaline cartilage fragments in the herniated disc material can suppress neovascularisation, and consequently reduction of the herniated disc material (7). Preoperative MR imaging of the lumbar disc herniations enables to draw relation between morphologic characteristics of the different types of lumbar disc herniations and the histologic composition of the operatively removed herniated disc material.

The aim of the study is to compare the data for histologic composition of herniated surgically removed disc material (especially the existence of granulation tissue as a part of natural healing mechanism) between different types of disc herniations and between patients of different age.

\section{Material and methods}

A total of 120 patients were included in the study (40 females and 80 males). Mean age of the patients was 46 years (range 35-65 years). All of the patients had low back pain and radiculopathy with duration more than four weeks. The patients' clinical symptomatology was adequate to the level and the site of the disc 
herniation. The patients with previous operative treatment of the disc herniation at the same lumbar level were not included in the study. Criteria for inclusion were clinical symptomatology for disc herniation, diagnostic evidence of the herniation with MR imaging, operative treatment and histopathological assessment of the surgically removed herniated disc material. Patients who met the inclusion criteria for the study were followed up for a 3-years period of time, while the MR imaging examination was performed as a preoperative procedure on the basis of the accepted indications for operative treatment of the disc herniations and patient's consent.

\section{MR imaging and disc herniations evaluation}

MR imaging examination was preformed with 1.5 T MR unit (Gyroscan T10- NT) with a spinal surface coil. The imaging protocol consisted of a sagittal T1-w Weighted spin-echo sequence (repetition time ms/echo time ms, 600/14; section thickness, 4 $\mathrm{mm}$; field of view, 280x280 mm; matrix, 240 x 256), sagittal T2Weighted turbo spin-echo sequence $(4,700 / 120$; section thickness, $4 \mathrm{~mm}$; intersection gap, $0.8 \mathrm{~mm}$; echo train length of 15), and a transverse $\mathrm{T} 2-\mathrm{w}$ Weighted turbo spin-echo sequence at one or multiple levels $(4,500 / 120$; section thickness, $4 \mathrm{~mm}$; intersection gap, $0.8 \mathrm{~mm}$; echo train length of 15 ; field of view, $200 \times 200$ mm; matrix $240 \times 256$ ). Depending on the pathologic condition transverse T1 weighted spin echo was preformed after administration of Gd-DTPA (Magnevist). The obtained MR images were assessed in consensus by two radiologists. Disc herniations were evaluated and classified as protrusion and extrusions based on the morphologic criteria accepted by the Nomenclature Committee of the North American Spine Society (8). Extrusions can be defined as suligamentous and trans or extraligamentous herniations, according to the position of the displaced disk material in relation to the posterior longitudinal ligament The posterior longitudinal ligamentous complex (PLLC) divides the epidural space into the perithecal space and the anterior epidural space, where displaced disk material can be entrapped. Posterior midline herniations seldom rupture the PLLC and are generally subligamentous. The lateral membranes are less resistant and transligamentous herniations are usually large posterolateral herniations. Migrated disc herniations are characterized by displacement of disk material away from the parent disc, either in the central spinal canal at the disk level, or above or below the disk level. In the cranio-caudal direction, disk migration was labeled by referring to the height of a vertebral body, divided in thirds. Large migrated disc herniations at the discal level are those that produce moderate and severe ( $>$ than one third) canal compromise (8). In our study on the basis of their morphology, containment, relation to the PLL, volume and migration of the herniated disc material, disc herniations were divided in to two groups. The first group comprised of nonmigrated herniations with herniated disc material remaining at the discal level like protrusions and contained subligamentous extrusions (mostly posterior midline herniations at the discal level that produce mild canal compromise). Posterior midline disc extrusions unless very large, usually remain entrapped under the deep layer of the PLL C or under the few intact outer anulus fibers joining with the PLL C to form a capsule. The second group included large migrated disc herniations at the discal level, like posterolateral disc extrusions with relatively large amount of displaced disc material away from the parent disc to the canal that produce moderate and severe canal compromise, foraminal disc herniations, free sequesters and far migrated disc extrusions cranially or caudally behind the posterior margins (more than one third) of the adjacent vertebral bodies.

\section{Histopathological findings}

After the operative treatment of the patients (microdiscectomy) surgically removed tissue samples were sent to the Institute of Pathology (Medical Faculty in Skopje). They were embedded in paraffin and evaluated by one pathologist. Slices $4 \mu \mathrm{m}$ thick were stained with hematoxylin -eosin. The criteria used in order to evaluate the presence of anulus fibrosus and nucleus pulposus were the following: anulus fibrosus is characterized with a presence of lamellae of fibrous bundles oriented in the same direction, separated by homogeneous or fibrillar matrix with small chondrocytes arranged in rows. Nucleus pulposus has homogeneous cartilage or smoothly granular appearance without fascicular collagen fibers or may consist of thin bundles of collagen fibers, but in various directions separated by amorphous matrix (dominantly mucoid or myxoid) with cells irregularly arranged. If both annulus fibrosus and nucleus pulposus tissue were present, the classification was done on the basis of the prevailing tissue. According to the histologic composition of the herniated material four types of tissue composition were defined. Type I consisted of dominantly anulus fibrosus tissue, type II from dominantly nucleus pulposus tissue, type III with anular and nucleus pulposus tissue found in almost equal proportions. Type IV was characterized by dominant appearance of fibrous connective tissue without lamellar characteristics that cannot be classified as anulus fibrosus or nucleus pulposus tissue. The presence of hyaline cartilage from vertebral endplate characterized with chondrocytes with perinuclear halo and homogeneously structured matrix was evaluated too (Fig. 1). Small calcified areas were rarely visible. In most of the samples aggregates of chondrocytes like cells were evaluated as well as necrotic cells. Areas of granulation tissue were characterized with small vessels with thin layer of endothelial cells, mononuclear cells, macrophages and fibroblasts like cells (Figs 2 and 3). These areas were located in the peripheral portions of the discs. Granulation tissue invading the whole peripheral portion of the disc was not evaluated. Sometimes the cell population in these areas consisted of fibroblasts (Fig. 4).

\section{Statistical analysis}

To test the differences between two independent patient groups we used Mann - Whitney U test. The calculations were performed with software SPSS for Windows 13.0. Differences with $\mathrm{p}$ value less than .05 were considered to be statistically significant.

\section{Results}

\section{Histologic composition of herniated disc material in different} types of disc herniations

In 64 patients with herniations classified as protrusions and nonmigrated extrusions the most frequent histologic composition 


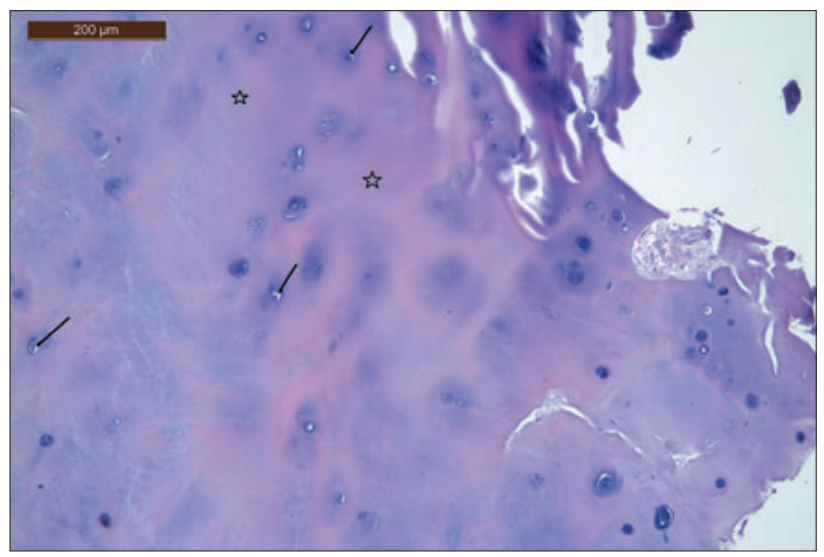

Fig. 1. Hyaline cartilage tissue in the herniated disc material with chondrocytes with perinuclear halo (arrows) and homogeneously structured matrix (stars).

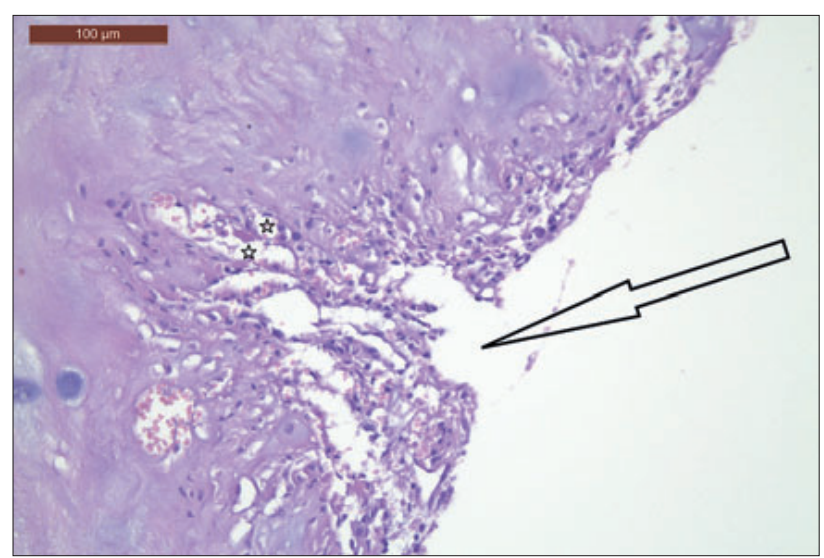

Fig. 2. Left arrow shows rupture with eosinophil precipitate (stars) in reparation in continuity with the disc tissue.

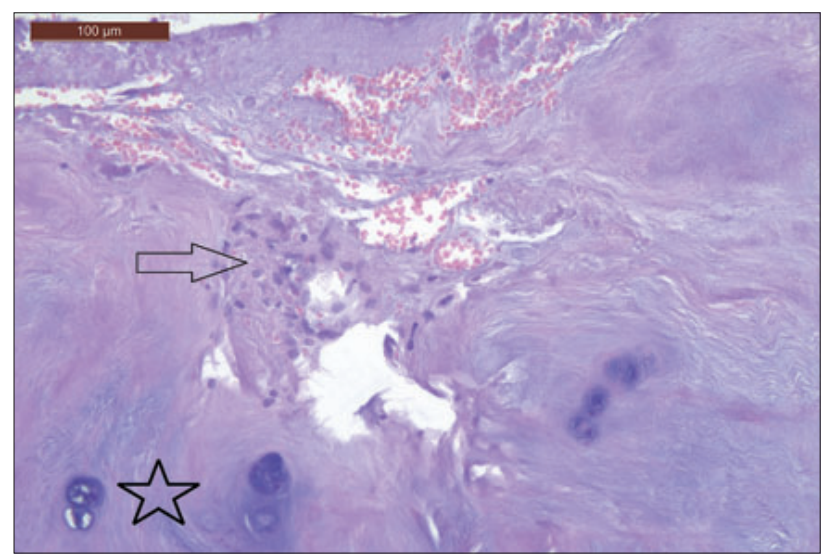

Fig. 3. Right arrow shows area of rupture in reparation in continuity with fibro hyaline cartilage tissue (star).

of the herniated material was dominant annulus fibrosus composition, in $50 \%$ of these herniations. In 12 herniations (19\%) nucleus pulposus tissue was predominant, while in $8(12 \%)$ anulus and nucleus pulposus were present in almost equal proportions. Twelve herniations had dominant fibrous tissue composition that

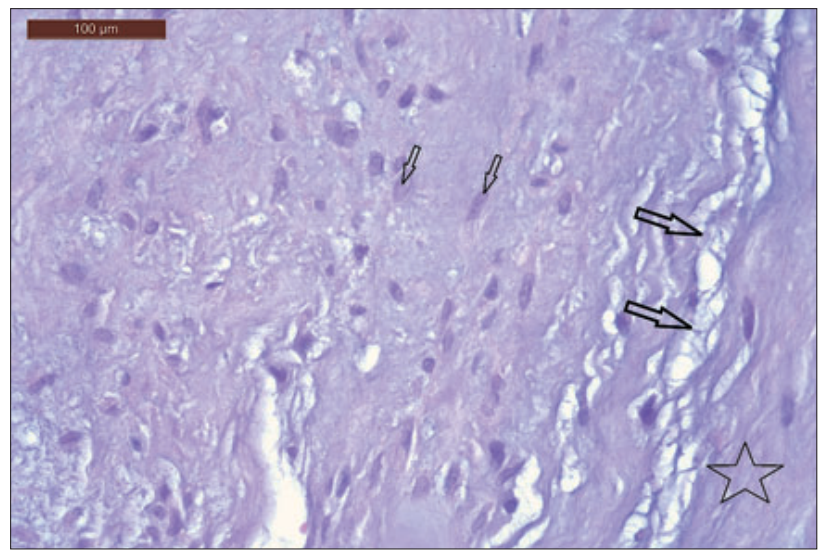

Fig. 4. Fibrous collagenous structured matrix (stars), mucoid degeneration (right arrow) and fibroblasts (down arrows) among irregularly arranged cells.

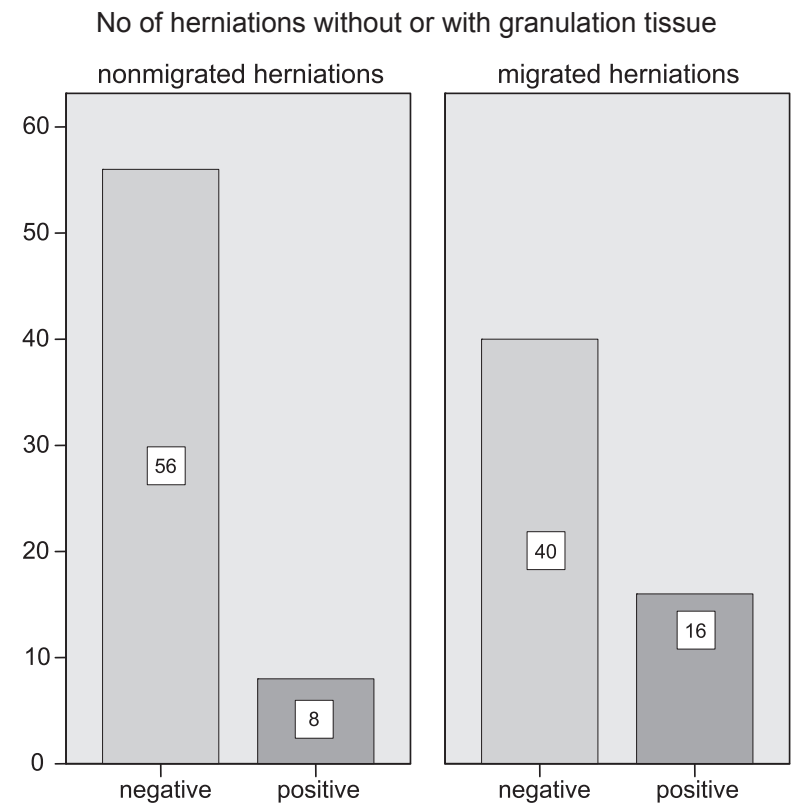

Fig. 5. Number of herniations with or without granulation tissue in nonmigrated and migrated disc herniations. The number of disc herniations with granulation tissue increased among large migrated disc herniations.

cannot be classified as anulus fibrosus or nucleus pulposus. In 56 patients with large transligamentous and migrated extrusions the tissue composition changed with slight decrease of prevailing anulus fibrosus composition, in 20 (36\%) herniations. Dominant nucleus pulposus composition was found in 12 (21\%) herniations,. An increase was evaluated, although not significant in the number of herniations with annulus fibrosus and nucleus pulpsus composition, in $12(21 \%)$ herniations. In 12 (21\%) of migrated disc herniations fibrous connective tissue was predominant. The tissue composition showed some differences, although not significant between the different types of disc herniations. Hyaline cartilage fragments were present in 24 (37\%) of protrusions and contained extrusions, while higher percentage of herniations with 
No of heniations without or with predominant fibrous matrix
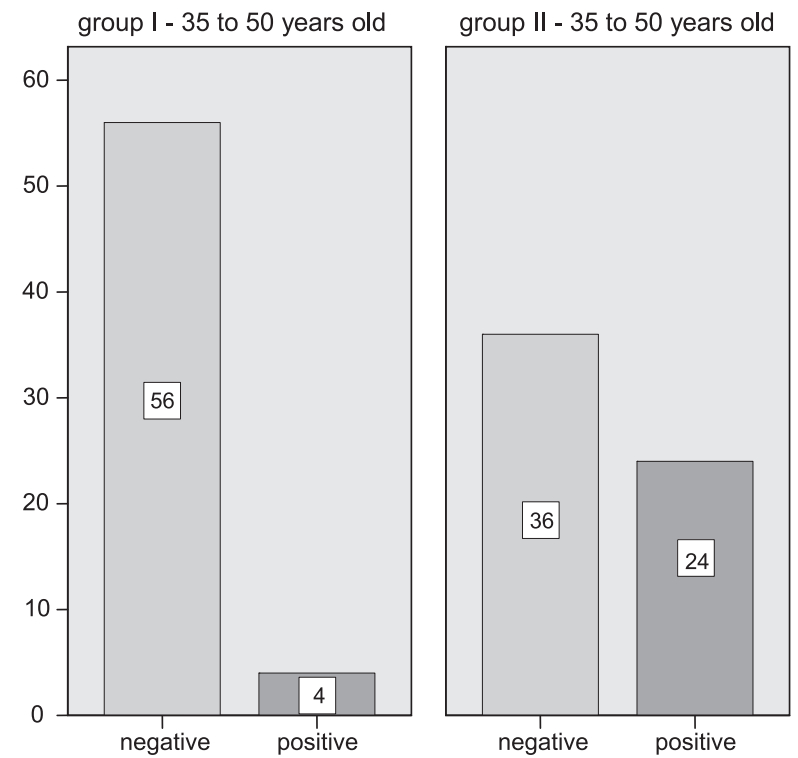

Fig. 6. Number of herniations with predominantly fibrous tissue composition in subjects of different age. The number of disc herniations with fibrous tissue composition increased in the older age group.

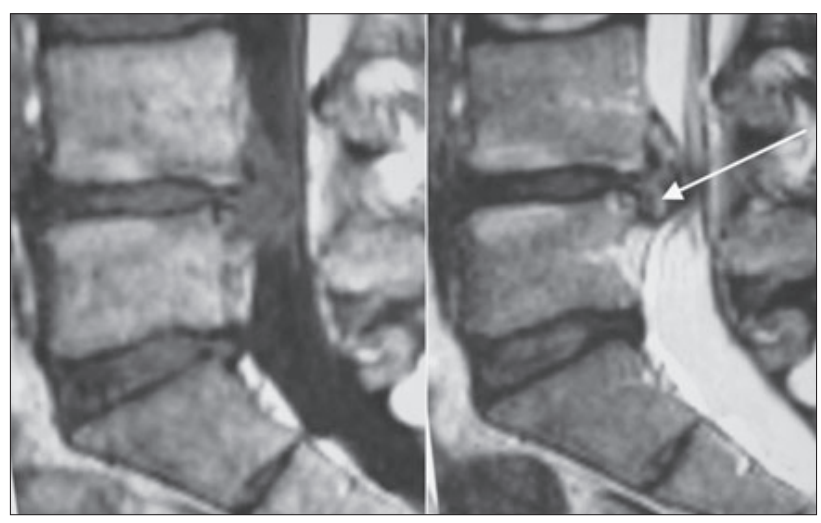

Fig. 7. Sagittal $T 1$ and $T 2$ weighted images of the lumbar spine in a 35 years old male patient. Sagittal T2- Weighted MR image shows a zone of higher signal intensity than the parent disc (arrow) in the migrated disc fragment behind the vertebral bodies of the two adjacent vertebrae. Extruded disc material consisted of annulus fibrosus, nucleus pulposus, hyaline cartilage from the vertebral endplate and area of granulation tissue.

cartilaginous fragments, $(64 \%)$ was found in patients with migrated extrusions ( $\mathrm{p}=0.04$, Mann-Whitney $\mathrm{U}$ test).

Areas of granulation tissue and neovascularisation were found in $8(12 \%)$ protrusions and contained extrusions, and in $16(29 \%)$ large migrated disc extrusions, with significant increase in the percentage of herniations with granulation tissue in the group of large migrated disc extrusions ( $\mathrm{p}=0.029$ Mann-Whitney U test) (Fig. 5).

\section{Histologic composition of the herniated disc material in subjects} of different age

In $20(33 \%)$ of 60 patients 35 to 50 years old, the composition of the herniated material was predominantly from anulus fibrosus,

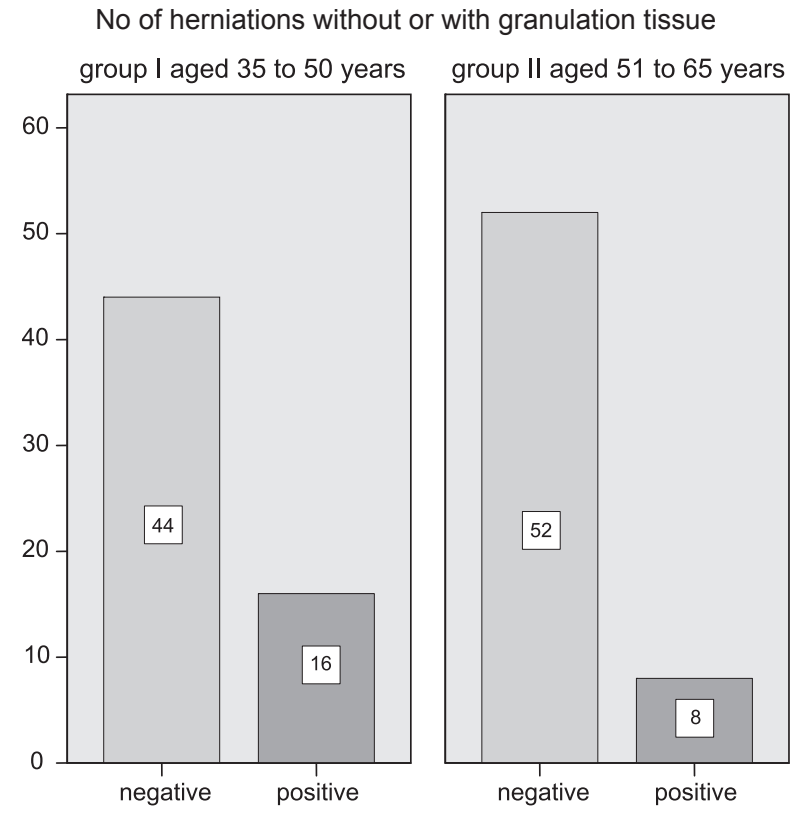

Fig. 8. Number of herniations with or without granulation tissue in subjects of different age. The number of disc herniations with granulation tissue decreased in the older age group.

in 20 patients nucleus pulposus was prevailing, while in $16(27 \%)$ patients anulus and nucleus pulposus were present in almost equal proportions. Only 4 (6\%) patients had prevailing fibrous tissue composition. In 60 patients 51 to 65 years old, the tissue composition of the herniated material changed with a slight increase of predominantly anulus fibrosus composition, in 28 (47\%) herniations, while in only 4 (7\%) prevailed dominantly nucleus pulposus composition with significant decrease in older patients $(p=0.000$, Mann-Whitney U test). A significant decrease was found in the herniations (7\%) with anulus fibrosus and nucleus pulposus composition as well ( $p=0.03$, Mann-Whitney $U$ test). The number of herniations with predominantly fibrous tissue composition significantly increased among the elderly patients, 24 (40\%) herniations belonged to type IV composition ( $p=0.000$, Mann-Whitney U test). The increase of this type of tissue composition in the elderly was a result of the previous fibrous degenerative changes in the intervertebral discs (Fig. 6).

Hyaline cartilage fragments were present in high percentage ( $40 \%$ ) of patients aged 35 to 50 years, but significantly higher percentage of herniations with cartilaginous fragments $(60 \%)$ was found in patients aged 51 to 65 years $(p=0.029$, Mann-Whitney test). Most of the herniations ( $53 \%$ ) with cartilaginous fragments consisted of predominantly annulus fibrosus tissue. Small calcified areas were found in 12 herniations in subjects aged $>50$ years.

Areas of granulation tissue and neovascularisation were found in $16(27 \%)$ of patients aged $\leq 50$. Some of these herniations were presented on sagittal $\mathrm{T} 2 \mathrm{~W}$ images with a higher intensity signal zone than the parent nucleus pulposus in the herniated tissue surrounded by a rim of dark signal (Fig. 7). In 8 (13\%) patients aged $>50$ a presence of granulation tissue in the herniated disc material was observed. The number of herniations with 
granulation tissue decreased, although not significantly with advancing age (Fig. 8).

Most of the herniations with granulation tissue were composed both of anulus fibrosus and nucleus pulposus tissue (50\%). Nucleus pulposus tissue was predominant constituent in $17 \%$ of these herniations. Hyaline cartilage fragments were seen in a high percentage $(83 \%)$ of the herniations with granulation tissue areas.

\section{Discussion}

The histologic composition of the herniated disc material has been subject of investigation of many studies. The limitations of the analyses of histologic composition of the disc herniation may be a consequence of the inconsistent classification of lumbar disc herniations, different criteria used in histologic interpretation and impossibility to define nucleus pulposus and annulus fibrosus like distinct entities in severely degenerated disc. The histologic composition of the examined herniated material from nonmigrated and migrated, large disc herniations showed no significant differences, while the histologic composition of the herniations from patients of different age was characterized with more significant differences. The percentage of the herniations with prevailing nucleus pulposus tissue decreased with increasing age. Harada and Nakahara also reported less maintained nucleus pulposus with increasing age (9). Due to the previous fibrocartilaginous transformation of the discs in the elderly, the percentage of the herniations with prevailing fibrous connective tissue without lamellar characteristics increased in these subjects. Higher percentages of herniations with cartilaginous fragments were found not only in large migrated herniations, but in older subjects, too. According to Tanaka avulsions occur more frequently in the inner or transitional zone of the annulus endplate surface (10). In our study $53 \%$ of the herniations with cartilaginous fragments consisted of predominantly annulus fibrosus tissue. In the study of Harada and Nakahara, disc fragments consisting of annulus fibrosus, cartilaginous end plate and bony fragments constituted $70 \%$ in the seventh and $80 \%$ in the eight decade. Willburger et al. reported higher percentages of cartilaginous end plate more frequently in patients $\geq 60$ years of age (11). Schmid reported avulsion-type disc herniations with hyaline cartilage material in nearly $50 \%$ of the patients (12). Our results confirm that the avulsion type of disc herniation are a predominant type of disc herniations in elderly patients, but they were also found in high percentage in patients $\leq 50$ years, mostly in large migrated disc herniations. In our study the areas of granulation tissue were almost always located in the peripheral parts of the herniated disc fragment. Granulation tissue with newly formed blood vessels, macrophages, fibroblasts and lymphocytes is probably related with the mechanism of resorption of the disc herniation and the vascular invasion of the disc tissue. The invasion of inflammatory cells and blood vessels in the herniated disc is a part of inflammatory reaction that may be a result of autoimmune response of antigens of nucleus pulposus or of the invasion of the herniated fragment treated as a foreign body undergoing inflammatory reaction. Both inflammation and proliferation of blood vessels lead to migration of phagocytes resulting in phagocytosis to remove the herniated disc material. Interaction between activated macrophages and disc tissue leads to generation of inflammatory cytokines and catabolic enzymes. These enzymes are responsible for the swelling of the proteoglycan molecules. Spontaneous regression as a result of enzymatic degradation is one of the hypothesis, the others are based on the retraction of the disc into the intervertebral space, and on the concept of dehydration with loss of water content of the herniated disc material. Many authors reported various characteristics of the herniations like factors related to the resorption process as penetration through the posterior longitudinal ligament (PLL), size of herniation, and existence of cartilage and anulus fibrosus tissue in the herniated material (13-16). Migrating and extruding type herniations have a higher tendency to decrease in size in followup studies $(13,14)$. Larger migrating type herniations demonstrate the greatest degree of resorption, so they regress more rapidly than smaller ones. Large herniations have a tendency to penetrate the capsula formed by anulus fibrosus and PLL and become exposed to systemic circulation in the epidural space.

In the study of Komori et al a higher tendency to decrease in size was noted for herniations that had more extensive rim enhancement in follow-up imaging (17). Gadolinium diethylenetriaminepentaacetic acid (Gd-DTPA) enhanced MRI could be used to evaluate the neovascularisation zone (17-19). Even without enhanced MRI examination with Gd-DTPA in some cases (with granulation tissue areas), on T2W images was evaluated a zone of higher signal intensity than the parent disc, surrounded completely or partially by a rim of dark signal in migrated herniations behind the vertebral bodies of the two adjacent vertebrae. In our study areas of granulation tissue were a more frequent finding in large migrated herniations, characterized with a high content of nucleus pulposus tissue in the herniated material. Normal nucleus pulposus has no vascular elements, so it cannot be recognized by the immune system. There are no inflammatory cells in the parent disc. In the situation of herniation, these tissues are recognized as an unknown antigen by the immune system and give rise to a host-immune response. When the nuclear material is exposed to the vascular environment of the epidural space, cellular mechanisms contribute to resorption (20). The margins of the extruded disc materials are infiltrated by mononuclear cells, and secretion of inflammatory mediators that are involved in the induction of angiogenesis is activated (21). Granulation tissue was also a more frequent finding in the herniated disc material from patients aged 35 to 50 years, thus our results also support the age dependence of HNP resorption. Histologic composition of the herniated material in elderly patients was characterized by prevailing fibrous and anulus fibrosus tissue and low content of nucleus pulposus. Hard and fibrous disc tissue may contribute to lower immunologic response and angiogenesis in older subjects. Some authors reported that clinical improvement in conservatively treated disc herniations was not correlated with their reduction in size on MRI (22). The limitation of this study is a lack of information about the duration of the period the herniations had appeared before surgery. According to some authors granulation tissue areas are a common finding in herniated material from the patients in whom the herniations had appeared long time before surgery. 
In conclusion, the tissue composition of the herniated material showed more expressed differences between subjects of different age than between different types of disc herniations. Granulation tissue and neovascularisation were more frequent findings in large migrated disc herniations and in herniated disc material from patients aged 35 to 50 years. The histologic composition of the majority of disc herniations with granulation tissue areas was composed of anulus fibrosus and nucleus pulposus in almost equal proportions.

\section{References}

1. Autio AR, Karppinen J, Niinima"ki J, Ojala R, Kurunlahti M,Haapea M, Vanharanta H, Tervonen O. Determinants of spontaneus resorption of intervertebral disc herniations. Spine 2006; (31) 11: $1247-1252$

2. Komori K, Shinomia K, Nakai O. The natural history of herniated nucleus pulposus with radiculopathy. Spine 1996; 21: 225-229.

3. Saal JA, Saal JS, Richard JH. The natural history of lumbar intervertebral disc extrusion treated nonoperatively. Spine 1990; 15: 683-686.

4. Kraemer J. Operations on the intervertebral disc. 231-244. In: Kraemer $\mathrm{J}$ ed. Intervertebral disc diseases. $2^{\text {nd }}$ ed. New York, NY: Thieme, 1990.

5. Takada E, Takahashi M,Shimada K. Natural history of lumbar disc hernia with radicular leg pain: Spontaneous MRI changes of the herniated mass and correlation with clinical outcome. Journal of Orthopaedic Surgery 2001; 9 (1): 1-7.

6. Carreon LY, Ito D, Yamada M et al. Histologic changes in the disc after cervical spine trauma : evidence of disc apsorption. J Spinal Disord 1996; 9: 313-316.

7. Carreon LY, Ito D, Yamada M et al. Neovascularisation induced by annulus and its inhibition by cartilage end plate: its role in disc apsorption. Spine 1997; 22: 1429-1434.

8. Fardon DF, Milette PC. Nomenclature and Classification of Lumbar Disc Pathology. Spine 2001; 26 (5): E93-E113.

9. Harada Y, Nakahara S. A pathologic study of lumbar disc herniations in the elderly. Spine 1989; 14: 1020-4.

10. Tanaka M, Nakahara S, Inoue H. A pathologic study of discs in the elderly: separation between the cartilaginous endplate and the vertebral body. Spine 1993; 18: 1456-1462.
11. Willburger ER, Ehiosun KU, Kuhnen C, Kramer J, Schmid G. Clinical symptoms in lumbar disc herniations and their correlation to the histologic composition of the extruded disc material. Spine 2004; 29 (15): 1655-1661.

12. Schmid G, Witteler A, Willburger R, Kuhnen C, Jergas M, Koester O. Lumbar disc herniation: correlation of histologic findings with marrow signal intensity changes in vertebral endplates at MR imaging. Radiology 2004; 231: 352-358.

13. Ahn SH, Ahn MW, Byun WM. Effect of the transligamentous extension oflumbar disc herniations on their regression and the clinical outcome of sciatica. Spine $2000 ; 25$ : 475-80.

14. Komori H, Shinomiya K, Nakai $O$ et al. The natural history of herniated nucleus pulposus with radiculopathy. Spine 1996; 21: 225-229.

15. Delauche-Cavallier MC, Budet C, Laredo JD et al. Lumbar disc herniation. Computed tomography scan changes after conservative treatment of nerve root compression. Spine 1992; 17: 927-933.

16. Kokubun S, Sakurai M, Tanaka Y. Cartilaginous endplate in cervical disc herniation. Spine 1996; 21: 190-195.

17. Komori H, Okawa A, Haro $\mathbf{H}$ et al. Contrast-enhanced magnetic resonance imaging in conservative management of lumbar disc herniation. Spine 1998; 23: 67-73.

18. Autio RA, Karppinen J, Kurunlahti M et al. Gadolinium diethylenetriaminepentaaceticacid enhancement in magnetic resonance imaging in relation to symptoms and signs among sciatic patients: A cross-sectional study. Spine 2002; 27: 1433-1437.

19. Ross JS, Modic MT, Masaryk TJ et al. Assessment of extradural degenerative disease with Gd-DTPA-enhanced MR imaging: Correlation with surgical and pathologic findings. AJNR Am J Neuroradiol 1989; 10: 1243-1249.

20. Satoh K, Konno S, Nishiyama K. Presence and distribution of antigen-antibody complexes in the herniated nucleus pulposus. Spine 1999; 24( 19): 1980-1984.

21. Saal JS, Franson RC, Dobrow R et al. High levels of inflammatory phospholipase A2 activity in lumbar disc herniations. Spine 1990; 7: 674-678.

22. Kamanly A,Karaca-Acet G,Kaya A, Koc M,Yildirim H. Conventional physical therapy with lumbar traction; clinical evaluation and MR imaging for lumbar disc herniations. Bratisl Lek Listy 2010; 111 (10): $541-544$. 